A. Braverman and D. Kazhdan

Nagoya Math. J.

Vol. 184 (2006), 57-84

\title{
SOME EXAMPLES OF HECKE ALGEBRAS FOR TWO-DIMENSIONAL LOCAL FIELDS
}

\author{
ALEXANDER BRAVERMAN AND DAVID KAZHDAN
}

\section{Dedicated to G. Lusztig on the occasion of his 60th birthday}

\begin{abstract}
Let $\mathbf{K}$ be a local non-archimedian field, $\mathbf{F}=\mathbf{K}((t))$ and let $\mathbf{G}$ be a split semi-simple group. The purpose of this paper is to study certain analogs of spherical and Iwahori Hecke algebras for representations of the group $\mathbb{G}=G(\mathbf{F})$ and its central extension $\hat{\mathbb{G}}$. For instance our spherical Hecke algebra corresponds to the subgroup $G(\mathcal{A}) \subset G(\mathbf{F})$ where $\mathcal{A} \subset \mathbf{F}$ is the subring $\mathcal{O}_{\mathbf{K}}((t))$ where $\mathcal{O}_{\mathbf{K}} \subset \mathbf{K}$ is the ring of integers. It turns out that for generic level (cf. [4]) the spherical Hecke algebra is trivial; however, on the critical level it is quite large. On the other hand we expect that the size of the corresponding Iwahori-Hecke algebra does not depend on a choice of a level (details will be considered in another publication).
\end{abstract}

\section{$\S 1$. Introduction}

1.1. Let Let $\mathbf{K}$ be a local non-archimedian field and let $\mathbf{F}=\mathbf{K}((t))$. In this paper we shall actually assume that $\mathbf{K}=\mathbb{F}_{q}((x))$ though this is probably not necessary. Denote by $\mathcal{O}_{\mathbf{K}}$ the ring of integers of $\mathbf{K}$. Set also $\mathcal{O}_{\mathbf{F}}=\mathbf{K}[[t]], \mathfrak{m}_{\mathbf{F}}=t \mathcal{O}_{\mathbf{F}}, \mathcal{A}=\mathcal{O}_{\mathbf{K}}((t)), \mathbf{E}=\mathbb{F}_{q}((t))$ and $\mathcal{O}_{\mathbf{E}}=\mathbb{F}_{q}[[t]]$. We denote by Vect the category of vector spaces over $\mathbb{C}$ and by $\mathbb{V e c t}$ the category of pro-vector spaces over $\mathbb{C}$. Let now $G$ be a connected split reductive algebraic group (defined over $\mathbb{Z}$ ). Set $\mathbb{G}=G(\mathbf{F})$. The category of $\operatorname{Rep}(\mathbb{G})$ of representations of $\mathbb{G}$ was defined in [4]. The group $\mathbb{G}$ admits canonical central extension $\hat{\mathbb{G}}$ by means of $\mathbf{K}^{*}$ and we denote by $\operatorname{Rep}(\hat{\mathbb{G}})$ the category of its representations. For each $\kappa: \mathbf{K}^{*} \rightarrow \mathbb{C}^{*}$ we denote by $\operatorname{Rep}_{\kappa}(\mathbb{G})$ the full subcategory of of $\operatorname{Rep}(\hat{\mathbb{G}})$ on which the central $\mathbf{K}^{*}$ acts by $\kappa$.

Received September 27, 2005.

Revised November 3, 2006.

2000 Mathematics Subject Classification: 22E55, 22E67. 


\subsection{Cherednik algebra as a Hecke algebra}

In this paper we want to discuss some examples of "Hecke algebras" for the group $\hat{\mathbb{G}}$. One of such examples is discussed in detail in [8] and [4]. Namely, let us choose a Borel subgroup $B$ of $G$ defined over $\mathbf{K}$ and let $U$ denote its unipotent radical and choose a Cartan subgroup $T$ in $B$. Let I $\subset G\left(\mathcal{O}_{\mathbf{F}}\right)$ be the Iwahori subgroup consisting of all elements of $G\left(\mathcal{O}_{\mathbf{F}}\right)$ whose reduction mod $t$ lies in $B(\mathbf{K})$. Let also $\mathbf{I}^{0}$ denote the preimage of $U(\mathbf{K})$ under the map $\mathbf{I} \rightarrow B(\mathbf{K})$ and set $\mathbf{I}^{00}=T\left(\mathcal{O}_{\mathbf{F}}\right) \mathbf{I}^{0}$. Consider the functor

$$
\operatorname{Coinv}_{\mathbf{I}^{00}}: \operatorname{Rep}(\hat{\mathbb{G}}) \longrightarrow \mathbb{V} e c t
$$

sending every representation to its coinvariants with respect to $\mathbf{I}^{00}$. Let $\ddot{\mathbf{H}}$ denote the algebra of endomorphisms of this functor. It is shown in [5] that this algebra is naturally isomorphic to Cherednik's double affine Hecke algebra associated with $G$. This is an extension of a previous result of Kapranov (cf. [8]).

\subsection{The $\operatorname{ring} \mathcal{A}$ and the corresponding subgroups}

One of the purposes of the present paper is to try to understand the results of [8] in some "global" context. In other words, one would like to develop some kind of two-dimensional theory of automorphic forms. Some speculations about this are presented in the last section of this article; the main part of the paper is devoted to a discussion of certain "Hecke algebras" which are supposed to play the same role in this (not yet constructed) theory as the usual Hecke algebras of $p$-adic groups play in the theory of automorphic forms. In the theory of representations of the group $G(\mathbf{K})$ the Hecke algebras are attached to open compact subgroups of this group; very often such subgroups can be realized as subgroups of $G\left(\mathcal{O}_{\mathbf{K}}\right)$ of finite index. Given such a group $\Gamma$ we can consider the functor $\operatorname{Inv}_{\Gamma}: \operatorname{Rep}_{G(\mathbf{K})} \rightarrow$ Vect sending every representation $V$ to the subspace $V^{\Gamma} \subset V$ of $\Gamma$-invariants. (Note that since $\Gamma$ is compact we have $V^{\Gamma}=V_{\Gamma}$ where $V_{\Gamma}$ is the quotient space of $\Gamma$-coinvariants.) The corresponding Hecke algebra $H(G, \Gamma)$ can be defined as the algebra of endomorphisms of this functor. It turns out (cf. some explanations in Section 1.14) that in our case the relevant subgroups of $G(\mathbf{F})$ are of the following form. Let $\mathcal{A}=\mathcal{O}_{\mathbf{K}}((t))^{1}$. We have the natural homomorphism $\mathcal{A} \rightarrow \mathbb{F}_{q}((t))$ (reduction modulo $\mathfrak{m}_{\mathbf{K}}$ ). Hence we get the natural homomorphism $\eta: G(\mathcal{A}) \rightarrow G\left(\mathbb{F}_{q}((t))\right)=G(\mathbf{E})$. The subgroups

\footnotetext{
${ }^{1}$ It is easy to see that $\mathcal{A}$ (as a subring of $\mathbf{F}$ ) does not depend on the choice of $t$.
} 
$\Gamma \subset \mathbb{G}$ in which we shall be interested in in this paper are those which are equal to the inverse image of a closed subgroup of $G(\mathbf{E})^{2}$. More specifically we are going to concentrate on the following two examples:

1) $\Gamma=G(\mathcal{A})$.

2) $\Gamma=\mathbf{I}_{\mathcal{A}}^{00}$ where

$$
\mathbf{I}_{\mathcal{A}}^{00}=\eta^{-1}\left(T\left(\mathcal{O}_{\mathbf{E}}\right) U(\mathbf{E})\right) .
$$

We shall refer to the first case as the spherical case and to the second as the Iwahori case. It is easy to see that the central extension $\hat{\mathbb{G}}$ splits over $G(\mathcal{A})$. Since $G(\mathcal{A})$ is equal to it's commutator we may consider the group $G(\mathcal{A})$ as a subgroup of $\hat{\mathbb{G}}$.

\subsection{The spherical Hecke algebra}

Fix $\kappa: \mathbf{F}^{*} \rightarrow \mathbb{C}^{*}$ as above and assume that it is trivial on $\mathcal{O}_{\mathbf{F}}^{*}$. In this case we may write $\kappa(x)=q^{c v(x)}$ where $c \in \mathbb{C}$. In this case we shall write $\operatorname{Rep}_{c}(\hat{\mathbb{G}})$ instead of $\operatorname{Rep}_{\kappa}(\hat{\mathbb{G}}) . \operatorname{Let}_{\operatorname{Inv}_{c}}: \operatorname{Rep}_{c}(\hat{\mathbb{G}}) \rightarrow \mathbb{V e c t}$ be the functor of invariants with respect to $G(\mathcal{A})^{3}$. Set

$$
\mathcal{H}_{c}^{\mathrm{sph}}=\operatorname{End}\left(\operatorname{Inv}_{c}\right) .
$$

In other words, $\mathcal{H}_{c}^{\mathrm{sph}}$ is the universal algebra acting on the (pro)space of $G(\mathcal{A})$-invariants in any representation of level $c$.

Remarks. 1. In this case the functor of invariants no longer coincides with the functor of coinvariants. It turns out that we have to use the former (it is not difficult to see that the latter is almost always trivial).

2. One can also study another version of the "double spherical Hecke algebra" when the subgroup $G(\mathcal{A})$ is replaced by $G\left(\mathcal{O}_{\mathbf{F}}\right)$ (and the functor of invariants is replaced by the functor of coinvariants). This is somewhat simpler but seems to be less relevant for "global" purposes discussed in Section 1.14.

Conjecture 1.5. (1) For any $c$ the algebra $\mathcal{H}_{c}^{\mathrm{sph}}$ is commutative.

(2) One has $\mathcal{H}_{c}^{\mathrm{sph}}=\mathbb{C}$ unless $q^{c+h^{\vee}}$ is a root of unity; here $h^{\vee}$ denotes the dual Coxeter number of $G$.

\footnotetext{
${ }^{2}$ One can consider a more general class of subgroups considering reductions modulo higher powers of $\mathfrak{m}_{\mathbf{K}}$. However, we are not going to do it in this paper.

${ }^{3}$ Since we are dealing with pro-vector spaces the notion of invariants is a bit tricky (cf. Section 3.1).
} 
Remark. The reader should compare this conjecture with the corresponding (known) statements for affine Lie algebras. For example the ana$\log$ of 1) for affine Lie algebras is a very general statement which is proved using the theory of vertex operator algebras. It seems that in our case one has to develop some sort of similar theory in order to prove such statements.

\subsection{The case of critical level}

The size of the algebra $\mathcal{H}_{c}^{\text {sph }}$ changes drastically when $q^{c+h^{\vee}}$ is a root of unity. In this paper we shall actually restrict ourselves to the critical level, i.e. to the case when $c=-h^{\vee}$ (in this case we shall write $\mathcal{H}_{\text {crit }}^{\text {sph }}$ instead of $\mathcal{H}_{-h^{\vee}}^{\mathrm{sph}}$ ). While we still can't show that in this case the algebra is commutative, we can construct a very large commutative subalgebra in it. This is done as follows. Let $\mathcal{B}=\mathcal{O}_{\mathbf{F}} \cap \mathcal{A}=\mathbb{F}_{q}[[x, t]]$. Let us consider the scheme $\Delta=\operatorname{Spec}(\mathcal{B})$. By an irreducible curve in $\Delta$ we shall mean a proper principal prime ideal of $\mathcal{B}$. If such a curve $C$ is given by the equation $f=0$ for some $f \in \mathcal{B}$ (i.e. $f$ is a generator of the corresponding ideal) then we say that $C$ is good if the ring $\mathcal{B}$ is topologically generated by $t$ and $f$ over $\mathbb{F}_{q}$ (thus automatically we have $\left.\mathcal{B}=\mathbb{F}_{q}[[f, t]]\right)$. In other words $C$ is a smooth irreducible curve which intersects the curve $X:=\{t=0\}$ transversely. Let $\mathcal{C}$ denote the set of good curves. Let $\mathcal{R}$ be the free commutative $\mathbb{C}$-algebra whose generators are elements of $\mathcal{C}$. Let us also denote by $\Lambda$ the coweight lattice of $G$ and by $\Lambda_{+} \subset \Lambda$ the subsemigroup of dominant coweights.

THEOREM 1.7. The algebra $\mathcal{H}_{\text {crit }}^{\mathrm{sph}}$ contains $\mathcal{R}\left[\Lambda_{+}\right]$as a subalgebra.

In this section we give a rough idea of the proof of Theorem 1.7 (which will also explain why the critical level is special) and provide details later in the paper. The theorem says basically that given any $C \in \mathcal{C}$ we may construct an embedding $\alpha_{C}: \mathbb{C}\left[\Lambda_{+}\right] \hookrightarrow \mathcal{H}_{c r i t}^{\text {sph }}$ and that their images commute for different curves $C$. In other words, given $C$ as above we need to construct the corresponding element $\alpha_{C}(\lambda) \in \mathcal{H}_{\text {crit }}^{\text {sph }}$ for each $\lambda \in \Lambda_{+}$such that for any $\lambda, \mu \in \Lambda_{+}$we have

$$
\alpha_{C}(\lambda) \alpha_{C}(\mu)=\alpha_{C}(\lambda+\mu)
$$

and such that for any two curves $C, C^{\prime}$ and any $\lambda, \mu \in \Lambda_{+}$the elements $\alpha_{C}(\lambda)$ and $\alpha_{C^{\prime}}(\mu)$ commute.

Let $C$ be given by the equation $f=0$. Choose a maximal torus $T$ in $G$ and set $f^{\lambda}=\lambda(f)$. Let $\mathbb{V}$ be a representation of $\hat{\mathbb{G}}$ of some level $c$. We need 
to construct an operator $\alpha_{C}^{\mathbb{V}}: \mathbb{V}^{G(\mathcal{A})} \rightarrow \mathbb{V}^{G(\mathcal{A})}$. Assume for simplicity that $\mathbb{V}^{G(\mathcal{A})}$ is a vector space (and not a pro-space) so that we can talk about its elements. Then for any $v \in \mathbb{V}^{G(\mathcal{A})}$ we would like to write

$$
\alpha_{C}^{\mathbb{V}}(\lambda)(v)=\int_{G(\mathcal{A}) f^{\lambda} G(\mathcal{A}) / G(\mathcal{A})} g(v) d g
$$

For this formula to make sense we have to

a) interpret the integrant $g(v) d g$ as a measure on the set $G(\mathcal{A}) f^{\lambda} G(\mathcal{A}) /$ $G(\mathcal{A})$

b) to show that the corresponding integral is convergent.

Let us note that the expression $g(v)$ doesn't make sense on $\mathbb{G}$ but only on $\hat{\mathbb{G}}$; more precisely, $g(v)$ makes sense as $\mathbb{V}$-valued function on the $\mathbb{Z}$-torsor over $\mathbb{G}$ obtained from the $\mathbf{K}^{*}$-torsor $\hat{\mathbb{G}} \rightarrow \mathbb{G}$ by applying the homomorphism $\mathbf{K}^{*} \rightarrow \mathbb{Z}$. Also the shift by $1 \in \mathbb{Z}$ multiplies it by $q^{-h^{\vee}}$.

Denote by $\mathcal{T}$ the $-h^{\vee}$-th power of this $\mathbb{Z}$-torsor. Thus $g(v)$ makes sense as a $\mathbb{V}$-valued function on $\mathcal{T}$ so that $1 \in \mathbb{Z}$ acts by multiplication by $q$. For a) we remind some basics of the theory of integration on varieties over local fields such as $\mathbf{E}$. Let $Y$ be a smooth algebraic variety of $\mathbf{E}$ and let $\Omega_{Y}$ denote the line bundle of highest forms on $Y$. This line bundle defines a $\mathbf{E}^{*}$-torsor over $Y(\mathbf{E})$. By composing it with the valuation homomorphism $\mathbf{E}^{*} \rightarrow \mathbb{Z}$ we get a $\mathbb{Z}$-torsor over $Y(\mathbf{E})$. We denote by $\mathcal{T}_{Y}$ the dual $\mathbb{Z}$-torsor. Let $s$ be a locally constant function on $\mathcal{T}_{Y}$ (with values in any vector space $V$ ) on which the shift by $1 \in \mathbb{Z}$ acts by means of multiplication by $q$. Then it is well known that $s$ defines a $V$-valued measure on $Y(\mathbf{E})$ for which all open compact subsets are measurable. In particular, $\int_{K} s \in V$ makes sense where $K$ is any open compact subset of $X(\mathbf{E})$. In our case it is easy to see that we have an equality

$$
G(\mathcal{A}) f^{\lambda} G(\mathcal{A}) / G(\mathcal{A})=G(\mathbf{E}[[f]]) f^{\lambda} G(\mathbf{E}[[f]])
$$

The latter set is known to be the set of E-points of a smooth variety $\operatorname{Gr}_{G}^{\lambda}$ (the union of all $\mathrm{Gr}_{G}^{\lambda}$ 's is the affine Grassmannian $\operatorname{Gr}_{G}$ of $G$ - cf. Section 2). Moreover, the main property of the critical level will be the following:

Proposition 1.8. The restriction of $\mathcal{T}$ to $G(\mathcal{A}) f^{\lambda} G(\mathcal{A}) / G(\mathcal{A})$ is isomorphic to $\mathcal{T}_{\mathrm{Gr}_{G}^{\lambda}}$. 
So we see that the part a) is achieved and we can consider $g(v) d g$ as a measure on the set $G(\mathcal{A}) f^{\lambda} G(\mathcal{A}) / G(\mathcal{A})$. If the variety $\operatorname{Gr}_{G}^{\lambda}$ is proper (and thus the set $\operatorname{Gr}_{G}^{\lambda}(\mathbf{E})$ is compact) then the convergence of the above integral would be obvious. However, this happens only in some very limited number of cases, so theoretically some convergence issues may arise. However, we show in Section 2 that the above integral is always absolutely convergent.

Remarks. 1. Note that the algebra $\mathbb{C}\left[\Lambda_{+}\right]$is (abstractly) isomorphic to the usual "finite" spherical Hecke algebra $\mathcal{H}_{f}^{\text {sph }}$ for the group $G(\mathbf{K}){ }^{4}$. In Section 3.6 we make this isomorphism canonical by looking at the action of $\mathcal{H}_{\text {crit }}^{\text {sph }}$ in the space of $G(\mathcal{A})$-invariants in some particular representation. However, apart from the case of $G=G L(n)$ we can't compute this isomorphism explicitly.

2. We expect that the algebra $\mathcal{R}\left[\Lambda_{+}\right]$is dense in $\mathcal{H}_{\text {crit }}^{\text {sph }}$ in some sense. A precise conjecture is formulated in Section 3.12.

\subsection{A variant}

Here we want to discuss what happens when the group $G(\mathcal{A})$ is replaced by $G\left(\mathcal{O}_{\mathbf{F}}\right)$ (as was remarked above this case is somewhat easier but seems to be less relevant for global applications discussed below). In this case we should consider the functor

$$
\operatorname{Coinv}_{c}: \operatorname{Rep}_{c}(\hat{\mathbb{G}}) \longrightarrow \mathbb{V} e c t
$$

sending every representation to its coinvariants with respect to $G\left(\mathcal{O}_{\mathbf{F}}\right)$. We set

$$
\mathcal{H}_{c}^{\mathrm{sph}^{\prime}}=\operatorname{End}\left(\operatorname{Coinv}_{c}\right) .
$$

As before we believe that the following is true:

CONJECTURE 1.10. (1) The algebra $\mathcal{H}_{c}^{\mathrm{sph}^{\prime}}$ is commutative for every $c \in \mathbb{C}$.

(2) One has $\mathcal{H}_{c}^{\mathrm{sph}^{\prime}}=\mathbb{C}$ unless $q^{c+h^{\vee}}$ is a root of unity.

Let us write $\mathcal{H}_{c r i t}^{\mathrm{sph}^{\prime}}$ instead of $\mathcal{H}_{-h^{\vee}}^{\mathrm{sph}^{\prime}}$. Then we have the following result

TheOREM 1.11. There exists an embedding $\mathbb{C}\left[\Lambda_{+}\right] \hookrightarrow \mathcal{H}_{\text {crit }}^{\mathrm{sph}^{\prime}}$.

\footnotetext{
${ }^{4}$ Here the subscript "f" stands for "finite".
} 
The proof of Theorem 1.11 is basically a word-by-word repetition of the proof of Theorem 1.7 which is discussed in Section 3 except that the last part of the proof (Section 3.10) is not needed in the current case.

In fact, we believe that Theorem 1.11 may be strengthened in the following way.

Conjecture 1.12. The embedding in Theorem 1.11 is an isomorphism.

The reader should think of Conjecture 1.12 as an analog of the density Conjecture 3.13 .

One can also formulate the following Conjecture which implies both Conjecture 1.10 and Conjecture 1.12. Let $\ddot{\mathbf{H}}_{c}$ as before denote the modified double affine Hecke algebra as defined in [8] and let $Z\left(\ddot{\mathbf{H}}_{c}\right)$ denote its center. It follows easily from the results of [8] and [4] that one has a natural map $j_{c}: Z\left(\ddot{\mathbf{H}}_{c}\right) \rightarrow \mathcal{H}_{c}^{\mathrm{sph}^{\prime}}$.

Conjecture 1.13. The map $j_{c}$ is surjective for any $c$.

\subsection{Global dreams}

Here we would like to give some (very speculative) motivation for considering the above Hecke algebras. Let $S$ be a smooth surface over $\mathbb{F}_{q}$ and let $X \subset S$ be a smooth geometrically irreducible projective curve in $S$. We assume for simplicity that we also have a retraction $S_{X} \rightarrow X$ which is equal to identity on $X$. Let $S_{X}$ be the formal neighbourhood of $X$ in $S$ and by $S_{X}^{0}$ the formal punctured neighborhood. We define in Section 4 the set $\operatorname{Bun}_{G}\left(S_{X}^{0}\right)$ of isomorphism classes of $G$-bundles on $S_{X}^{0}$ (over $\mathbb{F}_{q}$ ) and a canonical $\operatorname{Pic}(X)\left(\mathbb{F}_{q}\right)$-torsor $\widehat{\operatorname{Bun}}_{G}\left(S_{X}^{0}\right)$ over the set $\operatorname{Bun}_{G}\left(S_{X}^{0}\right)$. Using the degree map $\operatorname{Pic}(X) \rightarrow \mathbb{Z}$ we obtain a $\mathbb{Z}$-torsor $\widetilde{\operatorname{Bun}_{G}}\left(S_{X}^{0}\right)$ over $\operatorname{Bun}_{G}\left(S_{X}^{0}\right)$. Let $W$ be a finite collection of closed points of $X$ as a scheme over $\mathbb{F}_{q}$. Denote by $S_{W}$ the formal neighborhood of $W$ in $S$ and set $S_{W}^{0}=S_{W} \cap S_{X}^{0}$. For every $w \in W$ we denote by $\mathbf{K}_{w}$ the local field of $w$ in $X$ and by $\mathbf{F}_{w}$ the corresponding 2-dimensional local field (the completion of $S_{X}^{0}$ along $\left.\operatorname{Spec} \mathbf{K}_{w}\right)$. We let

$$
\mathbf{F}_{W}=\prod_{w \in W} \mathbf{F}_{w} .
$$

Similarly let $\mathcal{A}_{w} \subset \mathbb{F}_{w}$ be the subring considered in Section 1.3 and let $\mathcal{O}_{S, w}$ denote the formal ring of $S$ at $w$. Clearly we have $\mathcal{O}_{S, w}=\mathcal{A}_{w} \cap \mathcal{O}_{\mathbf{F}_{w}}$ 
and we denote by $\mathcal{R}_{w}$ the corresponding free commutative algebra whose generators are good curves in $\operatorname{Spec}\left(\mathcal{O}_{S, w}\right)$. Let also

$$
\mathcal{A}_{W}=\prod_{w \in W} \mathcal{A}_{w} .
$$

Let us also consider the group $\mathbb{G}_{W}=G\left(\mathbf{F}_{W}\right)$. We also denote by $\widetilde{\mathbb{G}}_{W}$ the corresponding central extension of $\mathbb{G}_{W}$ by means of $\mathbb{Z}$. Let $\operatorname{Bun}_{G}\left(S_{X}^{0}, W\right)\left(\mathbb{F}_{q}\right)$ denote the set of isomorphism classes of the following data:

- A principal $G$-bundle $\mathcal{M}$ on $S_{X}^{0}$ defined over $\mathbb{F}_{q}$.

- A trivialization of $\mathcal{M}$ on $S_{W}^{0}$.

We denote by $\widetilde{\operatorname{Bun}_{G}}\left(S_{X}^{0}, W\right)$ the pull-back of the $\mathbb{Z}$-torsor $\widetilde{\operatorname{Bun}_{G}}\left(S_{X}^{0}\right) \rightarrow$ $\operatorname{Bun}_{G}\left(S_{X}^{0}\right)$ under the natural map $\operatorname{Bun}_{G}\left(S_{X}^{0}, W\right) \rightarrow \operatorname{Bun}_{G}\left(S_{X}^{0}\right)$. Note that the ring $\mathcal{A}_{W}$ is exactly the ring of regular functions on $S_{W}^{0}$. Thus it is clear that the group $G\left(\mathcal{A}_{W}\right)$ acts on $\operatorname{Bun}_{G}\left(S_{X}^{0}, W\right)$ by changing the trivialization of $\mathcal{M}$ on $S_{W}^{0}$.

The following lemma is proved in Section 4.

Lemma 1.15. The action of $G\left(\mathcal{A}_{W}\right)$ on $\operatorname{Bun}_{G}\left(S_{X}^{0}, W\right)$ extends canonically to an action of $\widetilde{\mathbb{G}}_{W}$ on $\widehat{\operatorname{Bun}}_{G}\left(S_{X}^{0}, W\right)$ in such a way that the central $\mathbb{Z} \subset \widetilde{\mathbb{G}}_{W}$ acts on $\widetilde{\operatorname{Bun}_{G}}\left(S_{X}^{0}, W\right)$ in the natural way.

We expect to be able to define some "correct" pro-space of functions $\mathcal{F}_{W}$ on $\widehat{\operatorname{Bun}_{G}}\left(S_{X}^{0}, W\right)$ which should be a representation of the group $\widetilde{\mathbb{G}}_{W}$. In particular, we should also have the pro-space $\mathcal{F}$ of functions on $\operatorname{Bun}_{G}\left(S_{X}^{0}\right)$ by taking $W$ to be the empty set. It can also be defined by

$$
\mathcal{F}=\mathcal{F}_{W}^{G\left(\mathcal{A}_{W}\right)} .
$$

For any $c \in \mathbb{C}$ we should also consider the spaces $\mathcal{F}_{W, c}$ and $\mathcal{F}_{c}$ of functions on which $1 \in \mathbb{Z}$ acts by means of multiplication by $q^{c}$. Generalizing (1.2), for every decomposition $W=W^{\prime} \coprod W^{\prime \prime}$ we should have

$$
\mathcal{F}_{W^{\prime}, c}=\mathcal{F}_{W, c}^{\mathbb{G}\left(\mathcal{A}_{W^{\prime \prime}}\right)}
$$

Now combining (1.3) with Theorem 1.7 we see that for every $u \notin W$ we get an action of $\mathcal{R}_{u}\left[\Lambda_{+}\right]$on $\mathcal{F}_{W,-h^{\vee}}$. Moreover, for different choices of $u$ 
the corresponding endomorphisms of $\mathcal{F}_{W,-h^{\vee}}$ commute with each other. In other words, each $\mathcal{F}_{W,-h \vee}$ is endowed with an action of the commutative algebra

$$
\bigotimes_{u \notin W} \mathcal{R}_{u}\left[\Lambda_{+}\right]
$$

One should think about this action as the "affine" analog of Hecke operators acting on the space of automorphic forms for the group $G$ associated with the global field of rational functions on $X$. It would therefore be very interesting to construct some examples of common eigen-functions of the above operators. We believe that some generalizations of Eisenstein series considered in [7] should provide such examples.

\subsection{Acknowledgements}

We would like to thank A. Beilinson, R. Bezrukavnikov, V. Drinfeld, D. Gaitsgory, D. Kaledin and S. Kumar for helpful discussions on the subject. We are also grateful to the anonymous referee for several very useful remarks. The first author is partially supported by the NSF grant DMS0300271.

\section{$\S 2$. Some results on singularities of affine Schubert varieties}

In this section we collect some facts about the canonical class of certain Schubert varieties that we shall need in the future. All the results of this section follow easily from [3] and [1]. In what follows we fix a ground field $k$ and set $\mathcal{K}=k((x))$ and $\mathcal{O}=k[[x]]$.

\subsection{The affine Grassmannian}

Let $G$ be a split semi-simple group over $k$ and set $\operatorname{Gr}_{G}=G(\mathcal{K}) / G\left(\mathcal{O}_{K}\right)$. It is known that $\mathrm{Gr}_{G}$ has a natural structure of a proper ind-scheme over $k$. More precisely, it is known that the orbits of the group $G(\mathcal{O})$ on $\operatorname{Gr}_{G}$ are parameterised by the elements of $\Lambda_{+}$. For each $\lambda \in \Lambda_{+}$we shall denote by $\mathrm{Gr}_{G}^{\lambda}$ the corresponding orbit and by $\overline{\mathrm{Gr}}_{G}^{\lambda}$ its closure $\mathrm{Gr}_{G}$. The following theorem is proved in [3] (cf. also [9] and [10] for the corresponding result in characteristic 0$)$.

Theorem 2.2. (1) Each $\overline{\mathrm{Gr}}_{G}^{\lambda}$ is a normal and Cohen-Macaulay projective variety over $k$.

(2) Each $\overline{\mathrm{Gr}}_{G}^{\lambda}$ has a resolution of singularities ${ }^{5}$ and for every such reso-

\footnotetext{
${ }^{5}$ Of course, this statement is not empty only if char $k>0$.
} 


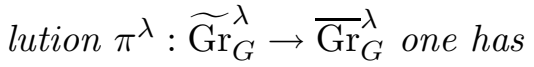

$$
R \pi_{*}^{\lambda}\left(\mathcal{O}_{\widetilde{\operatorname{Gr}}_{G}^{\lambda}}\right)=\mathcal{O}_{\overline{\operatorname{Gr}}_{G}^{\lambda}}
$$

(in other words $\overline{\mathrm{Gr}}_{G}^{\lambda}$ has rational singularities).

Remark. In fact the above theorem is proved in [3] in a slightly different context. Namely, let $\mathcal{F} l=G(\mathcal{K}) / I$ denote the affine flag variety as in [3], where $I$ is an Iwahori subgroup of $G(\mathcal{O})$ consisting of those elements of $G(\mathcal{O})$ whose reduction mod $t$ lies in a fixed Borel subgroup of $G$. The $I$ orbits of on $\mathcal{F l}$ are parametrised by the elements of the affine Weyl group $W_{\text {aff }}$; for every $w \in W_{\text {aff }}$ we let $\mathcal{F} l^{w}$ denote the corresponding orbit and by $\overline{\mathcal{F l}}^{w}$ its closure in $\mathcal{F l}$. The above theorem is proved in [3] for the varieties $\overline{\mathcal{F l}}^{w}$ rather than $\overline{\mathrm{Gr}}_{G}^{\lambda}$. However, it is easy to deduce the statement for $\overline{\mathrm{Gr}}^{\lambda}$, $\mathrm{s}$ from the statement for $\overline{\mathcal{F l}}^{w}$ 's. Namely, we have the natural proper smooth projection $p: \mathcal{F l} \rightarrow \mathrm{Gr}_{G}$. It is well-known that for every $\lambda \in \Lambda_{+}$there exist two elements $w_{\lambda}, w^{\lambda} \in W_{\text {aff }}$ such that: 1) The projection $p$ maps $\overline{\mathcal{F l}}^{w_{\lambda}}$ to $\overline{\mathrm{Gr}}^{\lambda}$ and the resulting map is proper and birational. 2) $\overline{\mathcal{F l}} w^{w^{\lambda}}=p^{-1}\left(\overline{\mathrm{Gr}}_{G}^{\lambda}\right)$. It follows from 1) that any resolution of $\overline{\mathcal{F l}}^{w_{\lambda}}$ is automatically a resolution of $\overline{\mathrm{Gr}}_{G}^{\lambda}$; this shows that the latter variety has a resolution of singularities. Now given the existence of a resolution, all the properties claimed in Theorem 2.2 are properties of the singularities of $\overline{\mathrm{Gr}}_{G}^{\lambda}$; in particular, it is easy to see that they are equivalent under smooth base change. Hence 2) shows that it is enough to check them for $\overline{\mathcal{F} l} w^{\lambda}$.

\subsection{Line bundles on $\operatorname{Gr}_{G}$}

It is well-known (cf. [1] and [3]) that every finite-dimensional representation $V$ of $G$ gives rise to a (determinant) line bundle $\mathcal{L}_{V}$ on $\mathrm{Gr}_{G}$. In particular, we let $\mathcal{L}_{\mathfrak{g}}$ (here $\mathfrak{g}$ is the Lie algebra of $G$ ) denote the line bundle corresponding to the adjoint representation of $G$. We let $\mathcal{L}_{\text {crit }}$ be the "critical line bundle" on $\mathrm{Gr}_{G}$; by the definition this is the (unique) square root of $\mathcal{L}_{\mathfrak{g}}^{-1}$.

In fact, it is well-known (cf. [3]) that $\operatorname{Pic}\left(\operatorname{Gr}_{G}\right) \simeq \mathbb{Z}$. Under this identification the line bundle $\mathcal{L}_{\mathfrak{g}}$ is the $2 h^{\vee}$-th power of a generator of $\mathbb{Z}$; we shall denote this generator by $\mathcal{D}$.

Let $\widehat{G}(\mathcal{K})$ be the central extension of $G(\mathcal{K})$ by means of $k^{*}$ constructed in the same way as the one discussed in Section 1.1 (with $k$ playing the role of $\mathbf{K}$ and $x$ playing the role of $t)$. Then $\mathcal{L}_{\text {crit }}$ is $\widehat{G}(\mathcal{K})$-equivariant in such a 
way that the central $k^{*}$ acts on the fibers of $\mathcal{L}_{\text {crit }}$ by means of the character $a \mapsto a^{-h^{\vee}}$. The following result from [1] is crucial for us:

THEOREM 2.4. For every $\lambda \in \Lambda_{+}$there is a canonical isomorphism

$$
\left.\mathcal{L}_{\text {crit }}\right|_{\mathrm{Gr}_{G}^{\lambda}} \simeq \Omega_{\mathrm{Gr}_{G}^{\lambda}}
$$

(Here, as in the Introduction, $\Omega_{\mathrm{Gr}_{G}^{\lambda}}$ denotes the bundle of highest forms on $\left.\mathrm{Gr}_{G}^{\lambda} \cdot\right)$

The next result is an easy corollary of Theorem 2.2 and Theorem 2.4.

TheOREM 2.5. (1) For every $\lambda \in \Lambda_{+}$the variety $\overline{\mathrm{Gr}}_{G}^{\lambda}$ is Gorenstein. Moreover, the canonical sheaf of $\overline{\mathrm{Gr}}_{G}^{\lambda}$ is isomorphic to $\left.\mathcal{L}_{\text {crit }}\right|_{\overline{\mathrm{Gr}}_{G}^{\lambda}}$. Abusing the notation we shall denote this sheaf by $\Omega_{\overline{\mathrm{Gr}}_{G}^{\lambda}}$.

(2) For any $\lambda \in \Lambda_{+}$let $\pi^{\lambda}:{\widetilde{\mathrm{Gr}_{G}}}_{G}^{\lambda} \rightarrow \overline{\mathrm{Gr}}_{G}^{\lambda}$ be any resolution of singularities. Then the identification between $\left(\pi^{\lambda}\right)^{*} \Omega_{\overline{G r}_{G}^{\lambda}}$ and $\Omega_{\widetilde{G r}_{G}^{\lambda}}$ that one has at the generic point of $\widetilde{\mathrm{Gr}}_{G}^{\lambda}$ comes from an embedding

$$
\left(\pi^{\lambda}\right)^{*} \Omega_{\overline{\mathrm{Gr}}_{G}^{\lambda}} \longleftrightarrow \Omega_{\widetilde{\mathrm{Gr}}_{G}^{\lambda}}
$$

(In the case char $k=0$ this implies that $\overline{\mathrm{Gr}}_{G}^{\lambda}$ has canonical singularities.)

Proof. Let us first prove (1). Let $\Omega_{\overline{G r}_{G}^{\lambda}}$ denote the GrothendieckSerre dualizing complex of $\overline{\mathrm{Gr}}_{G}^{\lambda}$ shifted by $-\operatorname{dim} \operatorname{Gr}_{G}^{\lambda}$ (so that it coincides with $\Omega_{\mathrm{Gr}_{G}^{\lambda}}$ on $\mathrm{Gr}_{G}^{\lambda}$ ). According to Theorem 2.2 the variety $\overline{\mathrm{Gr}}_{G}^{\lambda}$ is CohenMacaulay. Hence $\Omega{\overline{\mathrm{Gr}_{G}}}_{G}$ is in fact a sheaf (and not a complex of sheaves) which is automatically reflexive. Equivalently, one can reformulate this as follows. Let $j^{\lambda}: \operatorname{Gr}_{G}^{\lambda} \hookrightarrow \overline{\operatorname{Gr}}_{G}^{\lambda}$ denote the natural open embedding. Since $\overline{\mathrm{Gr}}_{G}^{\lambda}$ is normal and the complement to $\mathrm{Gr}_{G}^{\lambda}$ in $\overline{\mathrm{Gr}}_{G}^{\lambda}$ has codimension 2 we have $\Omega_{\overline{\mathrm{Gr}}_{G}^{\lambda}}=j_{*}^{\lambda} \Omega_{\mathrm{Gr}_{G}^{\lambda}}$. Now the normality of $\overline{\mathrm{Gr}}_{G}^{\lambda}$ also implies that

$$
\left.\mathcal{L}_{\text {crit }}\right|_{\overline{\mathrm{Gr}}_{G}^{\lambda}}=j_{*}^{\lambda}\left(\left.\mathcal{L}_{\text {crit }}\right|_{\mathrm{Gr}_{G}^{\lambda}}\right)=j_{*}^{\lambda} \Omega_{\mathrm{Gr}_{G}^{\lambda}}=\Omega_{\overline{\mathrm{Gr}}_{G}^{\lambda}} .
$$

Let us now prove (2). According to Theorem 2.2 we have $R \pi_{*}^{\lambda} \mathcal{O}_{\widetilde{\operatorname{Gr}}_{G}^{\lambda}}{ }^{\lambda}=\mathcal{O}_{\overline{\operatorname{Gr}}_{G}^{\lambda}}$. Applying the Grothendieck-Serre duality to both sides of this equality we 
get $R \pi_{*}^{\lambda} \Omega_{\widetilde{G r}_{G}^{\lambda}}=\Omega \frac{\lambda}{\operatorname{Gr}_{G}}$. By using the fact that the functor $\pi_{*}^{\lambda}$ is right adjoint to $\left(\pi^{\lambda}\right)^{*}$ we get the map $\left(\pi^{\lambda}\right)^{*} \Omega \frac{\lambda}{\overline{\mathrm{Gr}}_{G}} \rightarrow \Omega_{\widetilde{\mathrm{Gr}}_{G}^{\lambda}}$ which extends the natural identification of these line bundles at the generic point of $\widetilde{\mathrm{Gr}_{G}}{ }_{G}$.

\subsection{Application to integration}

Let now $\mathbf{E}$ be a local non-archimedian field and let $Y$ be a smooth variety over $\mathbf{E}$. Let $\mathcal{L}$ be a line bundle on $Y$. Denote by $\mathcal{L}_{\mathbf{E}^{*}}$ the corresponding $K^{*}$-torsor over $Y(\mathbf{E})$ and by $\mathcal{T}_{\mathcal{L}}$ the $\mathbb{Z}$-torsor obtained from $\mathcal{L}_{\mathbf{E}^{*}}$ by pushing-forward under the valuation map $\mathbf{E}^{*} \rightarrow \mathbb{Z}$. Set $\mathcal{S}(Y, \mathcal{L})$ to be the space of locally constant $\mathbb{C}$-valued functions $s$ on $\mathcal{T}_{\mathcal{L}^{-1}}$ satisfying the following properties:

1) The element $1 \in \mathbb{Z}$ acts on $s$ by means of multiplication by $q$;

2) The support of $s$ is equal to the preimage of a compact subset of $Y(\mathbf{E})$.

Note that every embedding $\mathcal{L}_{1} \rightarrow \mathcal{L}_{2}$ between two invertible sheaves on $Y$ gives rise to an embedding $\mathcal{S}\left(Y, \mathcal{L}_{1}\right) \rightarrow \mathcal{S}\left(Y, \mathcal{L}_{2}\right)$. Similarly, for a provector space $\mathbb{V}$ over $\mathbb{C}$ we may consider the pro-vector space $\mathcal{S}(Y, \mathcal{L}) \otimes \mathbb{V}$. Assume now that $Y$ is smooth and let $\mathcal{L}=\Omega_{Y}$ (in this case we shall write $\mathcal{T}_{Y}$ instead of $\mathcal{T}_{\Omega_{Y}^{-1}}$. Then it is well-known that we have a canonical integration functional

$$
\int_{Y}: \mathcal{S}\left(Y, \Omega_{Y}\right) \longrightarrow \mathbb{C} .
$$

Thus for a pro-vector space $\mathbb{V}$ we also get the integration map

$$
\int_{Y} \mathcal{S}\left(Y, \Omega_{Y}\right) \otimes \mathbb{V} \longrightarrow \mathbb{V} .
$$

Assume now that $Y$ is not necessarily smooth but satisfies the following conditions: 1) $Y$ is Gorenstein (we denote by $\Omega_{Y}$ the corresponding line bundle); 2) $Y$ has canonical singularities, i.e. there exists a resolution of singularities $\pi: \widetilde{Y} \rightarrow Y$ and an embedding $\pi^{*} \Omega_{Y} \rightarrow \Omega_{\widetilde{Y}}$ extending the natural identification of these line bundles at the generic point of $\widetilde{Y}$. Then we claim that the integration map $\int_{Y}$ as above is still well-defined. Indeed, it follows from condition 2) that every $s \in \mathcal{S}\left(Y, \Omega_{Y}\right)$ gives rise to some $\widetilde{s} \in \mathcal{S}\left(\widetilde{Y}, \Omega_{\widetilde{Y}}\right)$ and we define

$$
\int_{Y} s=\int_{\widetilde{Y}} \widetilde{s}
$$


It is clear that $\int_{\widetilde{Y}} \widetilde{s}$ does not depend on a choice of a resolution $\widetilde{Y}$ since in fact this integral is equal to the integral of $s$ over the smooth part of $Y$ (the existence of $\tilde{Y}$ with the above properties shows that this integral is absolutely convergent). So, for any pro-vector space and any $\lambda \in \Lambda_{+}$we have a well-defined integration map

$$
\int_{\overline{\mathrm{Gr}}_{G}^{\lambda}}: \mathcal{S}\left(\overline{\mathrm{Gr}}^{\lambda}, \mathcal{L}_{c r i t}\right) \otimes \mathbb{V} \longrightarrow \mathbb{V} .
$$

Sometimes we shall write $\int_{\mathrm{Gr}_{G}^{\lambda}}$ instead of $\int_{\overline{\mathrm{Gr}}_{G}^{\lambda}}$.

\section{$\S 3$. The Hecke algebras}

In this section we prove Theorem 1.7 and discuss some corollaries of it. First, let us discuss the functor of invariants in more detail.

\subsection{The functor $\operatorname{Inv}_{c}$}

Since we are dealing with pro-vector spaces let us first explain what we mean by the functor $\operatorname{Inv}_{c}$ of $G(\mathcal{A})$-invariants on the category $\operatorname{Rep}_{c}(\hat{\mathbb{G}})$. In [4] the authors explain the for any $\mathbb{V} \in \operatorname{Rep}(\hat{\mathbb{G}})$ and any subgroup $H \in$ $\hat{\mathbb{G}}$ one can define a functor from $\mathbb{V} e c t$ to Vect which is supposed to be represented by $\mathbb{V}^{H}$. However, this functor is representable only if certain conditions are satisfied. In this sub-section we want to check that these conditions are satisfied in the case of the subgroup $G(\mathcal{A}) \subset \hat{\mathbb{G}}$. In fact this is not necessary for the rest of this section. Namely in the next subsection we are going to construct some endomorphisms of $\mathbb{V}^{G(\mathcal{A})}$ (for every $\mathbb{V})$. For this purpose we shall only need the functorial definition $V^{G(\mathcal{A})}$. However, the fact that $V^{G(\mathcal{A})}$ actually exists as a pro-vector space shows the existence of the functor $\operatorname{Inv}_{c}: \operatorname{Rep}_{c}(\hat{\mathbb{G}}) \rightarrow \mathbb{V}$ ect and it still nice to know that the endomorphisms that we are going to construct will actually be endomorphisms of this functor.

Let us recall some definitions from [4].

Let us denote by $S_{0} t_{0}$ the category of finite sets. Let also

$$
\mathbf{S} e t=\operatorname{Ind}\left(\operatorname{Pro}\left(\operatorname{Set}_{0}\right)\right) ; \quad \mathbb{S} e t=\operatorname{Ind}(\operatorname{Pro}(\mathbf{S} e t)) .
$$

We have the natural functor $T: S_{0} t_{0} \rightarrow \mathbf{S} e t$. We shall need the functor

$$
\mathbb{T}: \mathbf{S} e t=\operatorname{Ind}\left(\operatorname{Pro}\left(\operatorname{Set}_{0}\right)\right) \longrightarrow \mathbb{S} e t=\operatorname{Ind}\left(\operatorname{Pro}\left(\operatorname{Ind}\left(\operatorname{Pro}\left(\operatorname{Set}_{0}\right)\right)\right)\right.
$$

which is defined by first applying $T$ to the "inner" Set $_{0}$ and then applying Ind(Pro) to both sides. 
Let $\mathbb{H}$ be an object of $\mathbb{S} e t$. In [4] the notion of action of $\mathbb{H}$ on a pro-vector space $\mathbb{V}$ is defined. Also if $\mathbb{H}$ is a group-like object of $\mathbb{S}$ et then the notion of a representation of $\mathbb{H}$ on a pro-vector space is defined and we also have a well defined category $\operatorname{Rep}(\mathbb{H})$ of pro-representations of $\mathbb{H}$. One has the functor triv : Vect $\rightarrow \operatorname{Rep}(\mathbb{H})$ corresponding to "trivial" representations. It is shown in [4] (cf. Proposition 2.10) that this functor always admits a left adjoint (which should be called the functor of coinvariants of $\mathbb{H}$ ). In addition, triv also admits a right adjoint functor if the following technical condition is satisfied:

(**) As an object of $\mathbb{S} e t$ the group $\mathbb{H}$ can be represented as a limit "lim" $X_{k}{ }^{6}$ where each $X_{k} \in \operatorname{Pro}(\mathbf{S} e t)$ is weakly strict (we refer the reader to [4] for the definition of this notion).

In our case let $\mathbb{H}=G(\mathcal{A})$. The $\operatorname{ring} \mathcal{A}$ naturally gives rise to an object of Set since

$$
\mathcal{A}=\lim _{\rightarrow} t^{-k} \mathcal{O}_{\mathbf{K}}[[t]]
$$

and the ring $\mathcal{O}_{\mathbf{K}}[[t]]$ is clearly a projective limit of finite sets. However, we are going to regard the ring $\mathcal{A}$ as an object in $\mathbb{S} e t$ by applying the functor $\mathbb{T}$ to the above construction. For any affine algebraic variety $X$ over $\mathcal{A}$ we may also regard $X(\mathcal{A})$ as an object of $\mathbb{S}$ et by embedding it into $\mathcal{A}^{n}$. Moreover, $X(\mathcal{A})$ always satisfies the condition (**) (it is enough to check this for $\mathcal{A}$ itself and in this case this is obvious).

Thus the group $G(\mathcal{A})$ may be regarded as a group-like object of the category $\mathbb{S} e t$ which satisfies $(* *)$. Hence the functor of $G(\mathcal{A})$-invariants is well-defined on the category $\operatorname{Rep}(G(\mathcal{A}))$ according to loc. cit. Also, the embedding $G(\mathcal{A}) \rightarrow \hat{\mathbb{G}}$ is an embedding of group-like objects of $\mathbb{S} e t$. Hence the restriction functor $\operatorname{Rep}_{c}(\hat{\mathbb{G}}) \rightarrow \operatorname{Rep}(G(\mathcal{A}))$ is well-defined. By composing these two functors we get the functor

$$
\operatorname{Inv}_{c}: \operatorname{Rep}_{c}(\hat{\mathbb{G}}) \longrightarrow \mathbb{V}
$$

of $G(\mathcal{A})$-invariants on the category $\operatorname{Rep}_{c}(\hat{\mathbb{G}})$.

\subsection{The construction of $\alpha_{C}$}

We want to define the homomorphism $\alpha_{C}$ by means of the formula (1.1). First of all, without loss of generality we may assume that $f=x$. Consider

\footnotetext{
${ }^{6}$ Here "lim" means that the limit is taken as an object of the corresponding Indcategory.
} 
the ring $\mathcal{B}=\mathbb{F}_{q}[[x, t]]$ and let $\mathbf{F}_{\mathcal{B}}$ denote its field of fractions. It is easy to see that the natural embedding $\mathcal{A}=\mathbb{F}_{q}[[x]]((t)) \hookrightarrow \mathbb{F}_{q}((t))[[x]]=\mathbf{E}[[x]]$ induces an isomorphism

$$
G(\mathcal{A}) x^{\lambda} G(\mathcal{A}) / G(\mathcal{A}) \stackrel{\sim}{\longrightarrow} G(\mathbf{E}[[x]]) x^{\lambda} G(\mathbf{E}[[x]])=\operatorname{Gr}_{G}^{\lambda}(\mathbf{E}) .
$$

We need now the following result which generalizes slightly Proposition 1.8. It follows from (3.1) that we have the natural identification

$$
\bigcup_{\mu \leq \lambda} G(\mathcal{A}) x^{\mu} G(\mathcal{A}) / G(\mathcal{A})=\overline{\mathrm{Gr}}_{G}^{\lambda}(\mathbf{E}) .
$$

The left hand side has a natural $\mathbf{K}^{*}$-torsor over it coming from the central extension $\hat{\mathbb{G}}$. As before we denote by $\mathcal{T}$ the $-h^{\vee}$-th power of the corresponding $\mathbb{Z}$-torsor.

Proposition 3.3. (1) Under the identification (3.2) the $\mathbb{Z}$-torsor $\mathcal{T}$ defined above goes over to $\mathcal{T}_{\overline{\mathrm{Gr}}_{G}^{\lambda}}^{\lambda}$.

(2) Let now $\mathbb{V}$ be a $\hat{\mathbb{G}}$-representation with the critical central charge. Then the formula $v \mapsto g(v)$ for

$$
g \in \bigcup_{\mu \leq \lambda} G(\mathcal{A}) x^{\mu} G(\mathcal{A}) / G(\mathcal{A})
$$

defines a linear map $\mathbb{V}^{G(\mathcal{A})} \rightarrow \mathcal{S}\left(\mathrm{Gr}_{G}^{\lambda}, \Omega_{\mathrm{Gr}_{G}^{\lambda}}\right) \otimes \mathbb{V}^{G(\mathcal{A})}$.

First of all, let us explain why Proposition 3.3 implies the construction

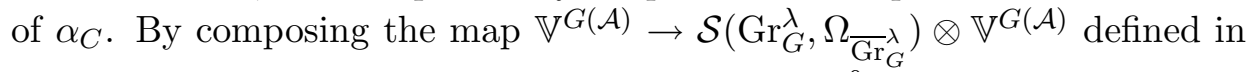
(2) of Proposition 3.3 it with the integration map $\int_{\mathrm{Gr}_{G}^{\lambda}}$ as in Section 2.6 we get a well defined linear map $\mathbb{V}^{G(\mathcal{A})} \rightarrow \mathbb{V}^{G(\mathcal{A})}$ which is clearly functorial in $\mathbb{V}$. In other words we get an element of $\mathcal{H}_{c r i t}^{\text {sph }}$ which by definition is equal to $\alpha_{C}(\lambda)$.

For the proof of Proposition 3.3 we need the following auxiliary result. Let $R$ denote the ring $\mathbb{F}_{q}\left[x, t, x^{-1}, t^{-1}\right]$. By the definition the ring $R$ is embedded naturally into the field $\mathbf{F}=\mathbb{F}_{q}((x))((t))$ as well as into the field $\mathbb{F}_{q}((t))((x))$. Hence the group $G(R)$ acquires two natural central extensions: one by means of $\mathbf{K}^{*}=\mathbb{F}_{q}((x))^{*}$ and the other by means of $\mathbf{E}^{*}=\mathbb{F}_{q}((t))^{*}$. By applying the valuation homomorphisms $\mathbf{K}^{*} \rightarrow \mathbb{Z}$ and $\mathbf{E}^{*} \rightarrow \mathbb{Z}$ we get two central extensions of $G(R)$ by means of $\mathbb{Z}$. Clearly, these two central extensions are interchanged by the automorphism of $G(R)$ induced by the automorphism of $R$ which interchanges $x$ and $t$. 
LEMMA 3.4. The above two central extensions are opposite to each other.

Proof. The proof follows from the following facts:

1) There exists a natural central extension

$$
1 \longrightarrow K_{2}(R) \longrightarrow \bar{G}(R) \longrightarrow G(R) \longrightarrow 1
$$

where $K_{2}(R)$ denotes the second $K$-group of $R$;

2) The two central extensions of $G(R)$ by $\mathbb{Z}$ are induced by the homomorphisms $K_{2}(R) \rightarrow \mathbb{Z}$ obtained by composition

$$
\begin{gathered}
K_{2}(R) \longrightarrow K_{1}\left(\mathbb{F}_{q}\left[x, x^{-1}\right]\right) \simeq \mathbb{F}_{q}^{*} \times \mathbb{Z} \longrightarrow \mathbb{Z} \\
\left(\operatorname{resp} . \quad K_{2}(R) \longrightarrow K_{1}\left(\mathbb{F}_{q}\left[t, t^{-1}\right]\right) \simeq \mathbb{F}_{q}^{*} \times \mathbb{Z} \longrightarrow \mathbb{Z}\right),
\end{gathered}
$$

where the first arrow comes from the Quillen isomorphism (cf. [11]) $K_{2}\left(A\left[t, t^{-1}\right]\right) \simeq K_{2}(A) \oplus K_{1}(A)$.

3) The above two homomorphisms $K_{2}(R) \rightarrow \mathbb{Z}$ are opposite to each other.

The first two statements follow from [2] and the third statement is proved in [11] (this is a special case of the existence of the Gersten resolution).

It is now clear that the assertion (1) of Proposition 3.3 follows from Lemma 3.4 combined with Theorem 2.4. From this the assertion (2) is straightforward.

It is easy to see that the homomorphisms $\alpha_{C}$ defined above together define a homomorphism $\mathcal{R}\left[\Lambda_{+}\right] \rightarrow \mathcal{H}_{\text {crit }}^{\text {sph }}$. Indeed, for this we have to verify the following two statements:

1) For any $C \in \mathcal{C}$ and $\lambda, \mu \in \Lambda_{+}$we have

$$
\alpha_{C}(\lambda) \cdot \alpha_{C}(\mu)=\alpha_{C}(\lambda+\mu) \text {. }
$$

2) For any $C, C^{\prime} \in \mathcal{C}$ and $\lambda, \mu \in \Lambda_{+}$the elements $\alpha_{C}(\lambda)$ and $\alpha_{C^{\prime}}(\mu)$ commute with each other.

First of all, if $C \neq C^{\prime}$ then statement 2) is obvious: in this case both $\alpha_{C}(\lambda) \cdot \alpha_{C^{\prime}}(\mu)$ and $\alpha_{C^{\prime}}(\mu) \cdot \alpha_{C}(\lambda)$ are given by integrals of the same measure over $\operatorname{Gr}^{\lambda}(\mathbf{E}) \times \operatorname{Gr}^{\mu}(\mathbf{E})$. Hence it is enough to check 1) (note that (3.4) implies 
that $\alpha_{C}(\lambda)$ and $\alpha_{C}(\mu)$ commute). For any field $k$ consider the ind-scheme $\mathrm{Gr}_{G} * \operatorname{Gr}_{G}$ whose set of $k$ points is equal $G(\mathcal{K}) \times_{G(\mathcal{O})} G(\mathcal{K}) / G(\mathcal{O})$ here as before we denote $\mathcal{K}=k((x))$ and $\mathcal{O}=k[[x]])$. We have the natural maps

$$
\begin{gathered}
p_{1}: G(\mathcal{K}) \times \operatorname{Gr}_{G} \longrightarrow \operatorname{Gr}, \quad p_{2}: G(\mathcal{K}) \times \operatorname{Gr}_{G} \longrightarrow \operatorname{Gr}_{G} \text { and } \\
m: \operatorname{Gr}_{G} * \operatorname{Gr}_{G} \longrightarrow \operatorname{Gr}_{G}
\end{gathered}
$$

which are defined by

$$
p_{1}\left(g_{1}, g_{2} \bmod G(\mathcal{O})\right)=g_{1} ; \quad p_{2}\left(g_{1}, g_{2} \bmod G(\mathcal{O})\right)=g_{2} \bmod G(\mathcal{O})
$$

and

$$
m\left(g_{1}, g_{2} \bmod G(\mathcal{O})\right)=g_{1} g_{2} \bmod G(\mathcal{O}) .
$$

We also have the natural projection $\pi: G(\mathcal{K}) \rightarrow \operatorname{Gr}_{G}$. For any $\lambda, \mu \in \Lambda_{+}$let us set $\mathrm{Gr}_{G}^{\lambda} * \mathrm{Gr}_{G}^{\mu}$ to be the image of $\left(\pi \circ p_{1}\right)^{-1}\left(\mathrm{Gr}_{G}^{\lambda}\right) \times p_{2}^{-1}\left(\mathrm{Gr}_{G}^{\mu}\right) \subset G(\mathcal{K}) \times \mathrm{Gr}_{G}$ in $\operatorname{Gr}_{G} * \operatorname{Gr}_{G}$. We also denote its closure by $\overline{\mathrm{Gr}}_{G}^{\lambda} * \overline{\mathrm{Gr}}_{G}^{\mu}$. The following result is well-known:

Lemma 3.5. The map $m$ maps $\mathrm{Gr}_{G}^{\lambda} * \mathrm{Gr}_{G}^{\mu}$ to $\overline{\mathrm{Gr}}_{G}^{\lambda+\mu}$.

Hence we also have the map $m: \overline{\mathrm{Gr}}_{G}^{\lambda} * \overline{\mathrm{Gr}}_{G}^{\mu} \rightarrow \overline{\mathrm{Gr}}_{G}^{\lambda+\mu}$ which is proper and birational. Thus (using the same notations as in (1.1) we get

$$
\begin{aligned}
\alpha_{C}^{\mathbb{V}} & (\lambda) \cdot \alpha_{C}^{\mathbb{V}}(\mu)(v) \\
& =\int_{g_{1} \in G(\mathcal{A}) f^{\lambda} G(\mathcal{A}) / G(\mathcal{A})} g_{1}\left(\int_{g_{2} \in G(\mathcal{A}) f^{\mu} G(\mathcal{A}) / G(\mathcal{A})} g_{2}(v) d g_{2}\right) d g_{1} \\
& =\int_{g \in \mathrm{Gr}_{G}^{\lambda}(\mathbf{E}) * \mathrm{Gr}_{G}^{\mu}(\mathbf{E})} g(v) d g=\int_{g \in \mathrm{Gr}_{G}^{\lambda+\mu}(\mathbf{E})} g(v) d g=\alpha_{C}^{\mathbb{V}}(\lambda+\mu)(v) .
\end{aligned}
$$

It remains to show that this homomorphism is an embedding. Before we proceed with this let us look at one example of the action of $\mathcal{R}\left[\Lambda_{+}\right]$on $\mathbb{V}^{G(\mathcal{A})}$ for some particular representation $\mathbb{V}$.

3.6. The homomorphism $\mathcal{H}_{c r i t}^{\mathrm{sph}} \rightarrow \mathcal{H}_{f}^{\mathrm{sph}}$

Let $\mathcal{H}_{f}^{\mathrm{sph}}$ denote the usual spherical Hecke algebra associated with the group $G$ and the local field $\mathbf{K}$. This algebra can be defined as follows. Let $\mathcal{S}\left(G\left(\mathcal{O}_{\mathbf{K}}\right) \backslash G(\mathbf{K})\right)$ be the space of $\mathbb{C}$-valued functions on $G\left(\mathcal{O}_{\mathbf{K}}\right) \backslash G(\mathbf{K})$ with finite support. This is a representation of the group $G(\mathbf{K})$ (acting on the right). Then

$$
\mathcal{H}_{f}^{\mathrm{sph}}=\operatorname{End}_{G(\mathbf{K})} \mathcal{S}\left(G\left(\mathcal{O}_{\mathbf{K}}\right) \backslash G(\mathbf{K})\right)
$$


Equivalently, $\mathcal{H}_{f}^{\mathrm{sph}}$ is the algebra of endomorphisms of the functor of $G\left(\mathcal{O}_{\mathbf{K}}\right)$ invariants on the category $\operatorname{Rep} G(\mathbf{K})$ of smooth representations of $G(\mathbf{K})$.

Let $G^{\vee}$ denote the Langlands dual group of $G$ (considered as an algebraic group over $\mathbb{C}$ ). Thus $\Lambda$ is the weight lattice of $G^{\vee}$ and $\Lambda_{+}$is the set of dominant weight of $G^{\vee}$. Recall that $\mathcal{H}_{f}^{\text {sph }}$ is canonically isomorphic to the complexified Grothendieck group of the category of finite-dimensional representations of $G^{\vee}$. In particular, $\mathcal{H}_{f}^{\text {sph }}$ has a basis $\left\{A_{\lambda}\right\}_{\lambda \in \Lambda_{+}}$where each $A_{\lambda}$ corresponds to the irreducible representation of $G^{\vee}$ with highest weight $\lambda$.

As is well-known the algebra $\mathcal{H}_{f}^{\text {sph }}$ is also isomorphic to the algebra of $G\left(\mathcal{O}_{\mathbf{K}}\right)$-biinvariant functions on $G(\mathbf{K})$ (the algebra structure is with respect to convolution; here we choose a Haar measure on $G(\mathbf{K})$ which is characterized by the property that the volume of $G\left(\mathcal{O}_{\mathbf{K}}\right)$ is equal to 1$)$. Since we have the natural identification

$$
G\left(\mathcal{O}_{\mathbf{K}}\right) \backslash G(\mathbf{K}) / G\left(\mathcal{O}_{\mathbf{K}}\right)=\Lambda_{+},
$$

it follows that $\mathcal{H}_{f}^{\text {sph }}$ has another basis $T_{\lambda}$ where each $T_{\lambda}$ is equal to the characteristic function of the corresponding double coset multiplied by $(-q)^{\left\langle\lambda, \rho^{\vee}\right\rangle} 7$. It is also well known that if we let $\rho^{\vee}$ denote the half-sum of the positive roots of $G$ then we have

$$
A_{\lambda}=\left(-q^{1 / 2}\right)^{\left\langle\lambda, \rho^{\vee}\right\rangle} T_{\lambda}+\text { linear combination of } T_{\mu} \text { with } \mu<\lambda^{8} .
$$

Let now $\Gamma_{1} \subset G\left(\mathcal{O}_{\mathbf{F}}\right)$ denote the first congruence subgroup (i.e. $\Gamma_{1}$ is the kernel of the natural "reduction mod $t$ " homomorphism $\left.G\left(\mathcal{O}_{\mathbf{F}}\right) \rightarrow G(\mathbf{K})\right)$. Let $\mathcal{S}\left(\hat{\mathbb{G}} / \Gamma_{1}\right)$ denote the Schwartz space ${ }^{9}$ of $\hat{\mathbb{G}} / \Gamma_{1}$ in the sense of [4] (equivalently, $\mathcal{S}\left(\hat{\mathbb{G}} / \Gamma_{1}\right)$ is the space of $\Gamma_{1}$-coinvariants in the Schwartz space $\mathcal{S}(\hat{\mathbb{G}})$ introduced in [4]). For $c \in \mathbb{C}$ we also denote by $\mathcal{S}_{c}\left(\hat{\mathbb{G}} / \Gamma_{1}\right)$ the coinvariants of $\mathbf{K}^{*}$ on $\mathcal{S}\left(\hat{\mathbb{G}} / \Gamma_{1}\right)$ with respect to the character defined by $c$. This is a representation of $\mathbb{G} \times G(\mathbf{K})$ where the first factor acts on the left and the second factor acts on the right. In particular, we may consider the space $\mathcal{S}_{c}\left(\hat{\mathbb{G}} / \Gamma_{1}\right)^{G(\mathcal{A})}$ of $G(\mathcal{A})$-invariants with respect to the left action. It has a natural action of $G(\mathbf{K})$. We have the natural embedding $\phi: G(\mathbf{K}) \rightarrow \Gamma_{1} \backslash \mathbb{G}$ which identifies the left hand side with $\Gamma_{1} \backslash G\left(\mathcal{O}_{\mathbf{K}}\right)$. Since the central extension splits canonically over $G\left(\mathcal{O}_{\mathbf{K}}\right)$ we get a well-defined restriction map

$$
\phi_{c}^{*}: \mathcal{S}_{c}\left(\Gamma_{1} \backslash \hat{\mathbb{G}}\right) \longrightarrow \mathcal{S}(G(\mathbf{K})) .
$$

\footnotetext{
${ }^{7}$ Here $\rho^{\vee}$ is the half-sum of all the positive roots of $G$.

${ }^{8}$ Here we say that $\mu<\lambda$ if the difference $\lambda-\mu$ is a sum of positive coroots.

${ }^{9}$ Recall that $\mathcal{S}\left(\hat{\mathbb{G}} / \Gamma_{1}\right)$ is actually a pro-vector space.
} 
Since the image of $G(\mathcal{A}) \cap G\left(\mathcal{O}_{\mathbf{K}}\right)$ is equal to $G\left(\mathcal{O}_{\mathbf{K}}\right)$ it follows that $\phi_{c}^{*}$ gives rise to the map

$$
\phi_{c, \mathcal{A}}^{*}: \mathcal{S}_{c}\left(\Gamma_{1} \backslash \hat{\mathbb{G}}\right)^{G(\mathcal{A})} \longrightarrow \mathcal{S}\left(G\left(\mathcal{O}_{\mathbf{K}}\right) \backslash G(\mathbf{K})\right)
$$

which is a homomorphism of right $G(\mathbf{K})$-modules.

Lemma 3.7. The map $\phi_{c, \mathcal{A}}^{*}$ defined above is an isomorphism.

Proof. The proof follows immediately from the following result.

LEMMA 3.8. For any connected linear reductive algebraic group $G$ one has $\mathbb{G}=G(\mathcal{A}) \cdot G\left(\mathcal{O}_{\mathbf{F}}\right)$.

This lemma is essentially contained in Appendix B of [6]. We include the proof for completeness (also, our proof is different from that in [6]).

Proof. The quotient $\mathbb{G} / G\left(\mathcal{O}_{\mathbf{F}}\right)$ is the set of $\mathbf{K}$-points of the Grassmannian $\operatorname{Gr}_{G}$. Since $\mathrm{Gr}_{G}$ is a proper ind-scheme, it follows that $\operatorname{Gr}_{G}(\mathbf{K})=$ $\operatorname{Gr}_{G}\left(\mathcal{O}_{\mathbf{K}}\right)$. This means that $G\left(\mathcal{O}_{\mathbf{K}}((t))\right)$ maps surjectively onto $\operatorname{Gr}_{G}(\mathbf{K})$, which is equivalent to the statement of Lemma 3.8.

Let now $h \in \mathcal{H}_{c}^{\mathrm{sph}}$. By the definition it gives rise to an endomorphism of $\mathcal{S}_{c}\left(\hat{\mathbb{G}} / \Gamma_{1}\right)^{G(\mathcal{A})}$ which commutes with every endomorphism of this space coming from an endomorphism of $\mathcal{S}_{c}\left(\hat{\mathbb{G}} / \Gamma_{1}\right)$ as a $\hat{\mathbb{G}}$-representation. In particular, it commutes with the right translations by elements of $G(\mathbf{K})$. Hence it follows from Lemma 3.7 that $h$ gives rise to an endomorphism of $\mathcal{S}\left(G\left(\mathcal{O}_{\mathbf{K}}\right) \backslash G(\mathbf{K})\right)$ which commutes with right translations by elements of $G(\mathbf{K})$. By (3.5) this is the same as an element of $\mathcal{H}_{f}^{\text {sph }}$. In this way we get a homomorphism

$$
\iota_{c}: \mathcal{H}_{c}^{\mathrm{sph}} \longrightarrow \mathcal{H}_{f}^{\mathrm{sph}}
$$

When $c=-h^{\vee}$ we shall denote $\iota_{c}$ by $\iota_{c r i t}$.

Lemma 3.9. For every $C \in \mathcal{C}$ we have

$$
\begin{aligned}
\iota_{\text {crit }} \circ \alpha_{C}(\lambda) & =\left(-q^{1 / 2}\right)^{\left\langle\lambda, \rho^{\vee}\right\rangle} A_{\lambda}+\text { linear combination of } A_{\mu} \text { 's with } \mu<\lambda \\
& =T_{\lambda}+\text { linear combination of } T_{\mu} \text { 's with } \mu<\lambda .
\end{aligned}
$$

In particular, $\iota_{\text {crit }} \circ \alpha_{C}$ is an isomorphism. 
Proof. First of all we want to show that $\iota_{\text {crit }} \circ \alpha_{C}(\lambda)$ lies in the span of $A_{\mu}$ with $\mu \leq \lambda$. This is equivalent to saying that $\iota_{\text {crit }} \circ \alpha_{C}(\lambda)$ lies in the span of $T_{\mu}$ with $\mu \leq \lambda$. It is clear that it is enough to assume that $C$ is given by the equation $x=0$.

To prove this assertion we must look at the support of $\iota_{\text {crit }} \circ \alpha_{C}(\lambda)$ considered as a $G\left(\mathcal{O}_{\mathbf{K}}\right)$-biinvariant function on $G(\mathbf{K})$. In other words, the statement is reduced to the following "set-theoretic" problem. We have the identification

$$
\Gamma_{1} \backslash \mathbb{G} / G(\mathcal{A})=G(\mathbf{K}) / G\left(\mathcal{O}_{\mathbf{K}}\right)=\operatorname{Gr}_{G}\left(\mathbb{F}_{q}\right) .
$$

Thus we obtain the map $\delta: \mathbb{G} / G(\mathcal{A}) \rightarrow \operatorname{Gr}_{G}\left(\mathbb{F}_{q}\right)$. We need to show that the image of $G(\mathcal{A}) x^{\lambda} G(\mathcal{A}) / G(\mathcal{A})$ under $\delta$ is contained in $\overline{\mathrm{Gr}}_{G}^{\lambda}\left(\mathbb{F}_{q}\right)$. Recall that we denote by $\mathbf{E}$ the field $\mathbb{F}_{q}((t))$ and that we have the natural identification

$$
G(\mathcal{A}) x^{\lambda} G(\mathcal{A}) / G(\mathcal{A})=\operatorname{Gr}_{G}^{\lambda}(\mathbf{E}) .
$$

Since $\operatorname{Gr}_{G}$ is ind-proper it follows that we have $\operatorname{Gr}_{G}(\mathbf{E})=\operatorname{Gr}_{G}\left(\mathcal{O}_{\mathbf{E}}\right)$ (here $\mathcal{O}_{\mathbf{E}}=\mathbb{F}_{q}[[t]]$ thus we have the well-defined "reduction $\bmod t$ " map

$$
G(\mathcal{A}) x^{\lambda} G(\mathcal{A}) / G(\mathcal{A})=\operatorname{Gr}_{G}^{\lambda}(\mathbf{E}) \rightarrow \operatorname{Gr}_{G}\left(\mathbb{F}_{q}\right) .
$$

It is easy to see that this map actually coincides with the restriction of $\delta$ to $G(\mathcal{A}) x^{\lambda} G(\mathcal{A}) / G(\mathcal{A})$. On the other hand, since $\overline{\mathrm{Gr}}_{G}^{\lambda}$ is proper, the above map actually lands in $\overline{\mathrm{Gr}}^{\lambda}\left(\mathbb{F}_{q}\right)$ which is what we had to prove.

To compute the coefficient of $T_{\lambda}$ in $\iota_{\text {crit }} \circ \alpha_{C}(\lambda)$ (which is the same as the coefficient of $A_{\lambda}$ up to the factor of $\left.\left(-q^{1 / 2}\right)^{\lambda, \rho^{\vee}}\right)$ we need to study the fibers of the map

$$
G(\mathcal{A}) x^{\lambda} G(\mathcal{A}) / G(\mathcal{A}) \cap \delta^{-1}\left(\operatorname{Gr}_{G}^{\lambda}\left(\mathbb{F}_{q}\right)\right) \longrightarrow \operatorname{Gr}_{G}^{\lambda}\left(\mathbb{F}_{q}\right) .
$$

Since $\operatorname{Gr}_{G}^{\lambda}\left(\mathbb{F}_{q}\right)$ is smooth, it follows from Hensel's lemma that each fiber is isomorphic to $\left(t \mathcal{O}_{\mathbf{E}}\right)^{\operatorname{dim} \mathrm{Gr}_{G}^{\lambda}}$ and its volume with respect to the measure coming from a differential form on $\operatorname{Gr}_{G}^{\lambda}$ defined over $\mathcal{O}_{\mathbf{E}}$ is equal to 1 . This finishes the proof.

Remark. Let $G=P G L(n)$. Then Lemma 3.9 describes the map $\iota_{c r i t}$ o $\alpha_{C}$ completely. Indeed, since the group $P G L(n)$ is of adjoint type, the semigroup $\Lambda_{+}$is generated by the fundamental coweights $\omega_{1}, \ldots, \omega_{n-1}$ and thus it is enough to describe all the $\iota_{\text {crit }}\left(\omega_{i}\right) \circ \alpha_{C}$. Also in this case for each $i=1, \ldots, n-1$ the set

$$
\left\{\mu \in \Lambda_{+} \mid \mu<\omega_{i}\right\}
$$


is empty. Hence $\iota_{\text {crit }} \circ \alpha_{C}\left(\omega_{i}\right)=A_{\omega_{i}}$. We don't know how to describe $\iota_{\text {crit }}$ for other groups.

\subsection{End of the proof}

We now want to show that the above homomorphism $\mathcal{R}\left[\Lambda_{+}\right] \rightarrow \mathcal{H}_{\text {crit }}^{\text {sph }}$ is an embedding. To do that it is sufficient to check the following: let $C_{1}, \ldots, C_{k}$ be pairwise distinct elements of $\mathcal{C}$ and let $\mu_{1}, \ldots, \mu_{k} \in \Lambda_{+}$. Consider the elements

$$
\alpha_{C_{1}}\left(\mu_{1}\right) \cdots \alpha_{C_{k}}\left(\mu_{k}\right) \in \mathcal{H}^{\mathrm{sph}}
$$

We need to show that all these elements are linearly independent.

For this we want to see how these elements act on the pro-space of $G(\mathcal{A})$-invariants for representations $\mathbb{V}_{n}=\mathcal{S}_{\text {crit }}\left(\hat{\mathbb{G}} / \Gamma_{n}\right)$ where $\Gamma_{n}$ denotes the $n$-th congruence subgroup (by definition $\Gamma_{n}$ is the kernel of the natural homomorphism $\left.G\left(\mathcal{O}_{\mathbf{F}}\right) \rightarrow G\left(\mathcal{O}_{\mathbf{F}} / \mathfrak{m}_{\mathbf{F}}^{n}\right)\right)$.

Let $G_{n}=G\left(\mathcal{O}_{\mathbf{F}} / \mathfrak{m}_{\mathbf{F}}^{n}\right)$ and let also $G_{n, \mathcal{O}}=G\left(\mathcal{O}_{\mathbf{K}}[[t]] / t^{n}\right)$. Then $G_{n}$ is a locally compact totally disconnected group and $G_{n, \mathcal{O}}$ is an open compact subgroup of it. Also we have the natural identification $\mathbb{V}_{n}^{G(\mathcal{A})}=$ $G_{n, \mathcal{O}} \backslash G_{n}$. Thus the image of $\mathcal{H}_{c r i t}^{\text {sph }}$ in $\operatorname{End}\left(\mathbb{V}_{n}^{G(\mathcal{A})}\right)$ embeds into the Hecke algebra $H\left(G_{n}, G_{n, \mathcal{O}}\right)$. In particular, we may think about the elements $\alpha_{C_{1}}^{\mathbb{V}_{n}}\left(\mu_{1}\right), \ldots, \alpha_{C_{k}}^{\mathbb{V}_{n}}\left(\mu_{k}\right)$ as $G_{n, \mathcal{O}}$-biinvariant functions on $G_{n}$. Thus it is enough to show that for any finite collection of elements of the type (3.6) their images in $H\left(G_{n}, G_{n, \mathcal{O}}\right)$ are linearly independent functions for $n$ large enough.

Let $f_{1}, \ldots, f_{k}$ be the equations of the curves $C_{1}, \ldots, C_{k}$ and set

$$
X_{n, C_{1}, \ldots, C_{k}}^{\lambda_{1}, \ldots, \lambda_{k}}=G_{n, \mathcal{O}} f_{1}^{\lambda_{1}} \cdots f_{k}^{\lambda_{k}} G_{n, \mathcal{O}} \subset G_{n}{ }^{10} .
$$

Lemma 3.11. (1) The element $\alpha_{C}^{\mathbb{V}_{n}}(\lambda)$ is supported on

$$
\bigcup_{\mu \leq \lambda} X_{n, C}^{\mu}
$$

and doesn't vanish on $X_{n, C}^{\lambda}$.

(2) Assume that the curves $C_{1}, \ldots, C_{k}$ are distinct. Then for any collection $\left(\left(C_{1}^{\prime}, \mu_{1}\right), \ldots,\left(C_{l}^{\prime}, \mu_{l}\right)\right)$ different from $\left(\left(C_{1}, \lambda_{1}\right), \ldots,\left(C_{k}, \lambda_{k}\right)\right)$ and for $n$ large enough the set $X_{n, C_{1}}^{\lambda_{1}} \cdots X_{n, C_{k}}^{\lambda_{k}}$ does not contain $X_{n, C_{1}^{\prime}, \ldots, C_{l}^{\prime}}^{\mu_{1}, \ldots, \mu_{l}}$.

\footnotetext{
${ }^{10}$ We shall always assume that $\lambda_{j} \neq 0$ unless $k=0$.
} 
(3) Fix a finite set of pairwise distinct collections $\left(\left(C_{1}, \lambda_{1}\right), \ldots,\left(C_{k}, \lambda_{k}\right)\right)$. The for $n$ large enough all the corresponding cosets $X_{n, C_{1}, \ldots, C_{k}}^{\lambda_{1}, \ldots, \lambda_{k}}$ are pairwise distinct.

Proof. The first assertion is proved in exactly the same way as Lemma 3.9. Let us prove (2) and (3). Let us "take the limit" $n \rightarrow \infty$. In other words, let us recall the notation $\mathcal{B}=\mathcal{O}_{\mathbf{F}} \cap \mathcal{A}=\mathbb{F}_{q}[[x, t]]$. For $\left(\left(C_{1}, \lambda_{1}\right), \ldots,\left(C_{k}, \lambda_{k}\right)\right)$ as above set

$$
X_{C_{1}, \ldots, C_{k}}^{\lambda_{1}, \ldots, \lambda_{k}}=G_{\mathcal{B}} f_{1}^{\lambda_{1}} \cdots f_{k}^{\lambda_{k}} G_{\mathcal{B}} \subset G\left(\mathcal{O}_{\mathbf{F}}\right)
$$

Then we have

$$
X_{C_{1}, \ldots, C_{k}}^{\lambda_{1}, \ldots, \lambda_{k}}=\lim _{\rightarrow} X_{n, C_{1}, \ldots, C_{k}}^{\lambda_{1}, \ldots, \lambda_{k}}
$$

and hence the assertions (2) and (3) of Lemma 3.11 follow from the following two statements:

a) Assume that the curves $C_{1}, \ldots, C_{k}$ are distinct. Then for any

$$
\left(\left(C_{1}^{\prime}, \mu_{1}\right), \ldots,\left(C_{l}^{\prime}, \mu_{l}\right)\right) \neq\left(\left(C_{1}, \lambda_{1}\right), \ldots,\left(C_{k}, \lambda_{k}\right)\right)
$$

the set $X_{C_{1}}^{\lambda_{1}} \cdots X_{C_{k}}^{\lambda_{k}}$ does not contain $X_{C_{1}^{\prime}, \ldots, C_{l}^{\prime}}^{\mu_{1}, \ldots, \mu_{l}}$.

b) The cosets $X_{C_{1}, \ldots, C_{k}}^{\lambda_{1}, \ldots, \lambda_{k}}$ are distinct for distinct collections $\left(\left(C_{1}, \lambda_{1}\right), \ldots\right.$, $\left.\left(C_{k}, \lambda_{k}\right)\right)$.

To prove a) and b) let us give a geometric interpretation of these statements. Recall that we denote $\Delta=\operatorname{Spec} \mathcal{B}$. Then it is easy to see that the set $G(\mathcal{B}) \backslash G\left(\mathcal{O}_{\mathbf{F}}\right) / G(\mathcal{B})$ naturally parametrises the following data:

1) Two principal $G$-bundles $\mathcal{F}_{1}, \mathcal{F}_{2}$ on $\Delta$;

2) An identification $\kappa$ between $\mathcal{F}_{1}$ and $\mathcal{F}_{2}$ defined outside the union of all the curves $C$ in $\Delta$ which are different from the curve $t=0$.

Under this identification, the coset $X_{C_{1}, \ldots, C_{k}}^{\lambda_{1}, \ldots, \lambda_{k}}$ lies in the subset $Y_{C_{1}, \ldots, C_{k}}^{\lambda_{1}, \ldots, \lambda_{k}}$ of all triples $\left(\mathcal{F}_{1}, \mathcal{F}_{2}, \kappa\right)$ as above such that $\kappa$ is well-defined outside the curves $C_{1}, \ldots, C_{k}$ and at the generic point of every $C_{j}$ the singularity of $\kappa$ is of type $\lambda_{j}$ (note, however, that in general $X_{C_{1}, \ldots, C_{k}}^{\lambda_{1}, \ldots, \lambda_{k}} \neq Y_{C_{1}, \ldots, C_{k}}^{\lambda_{1}, \ldots, \lambda_{k}}$ unless $k=1$ ). This immediately proves b) since the sets $Y_{C_{1}, \ldots, C_{k}}^{\lambda_{1}, \ldots, \lambda_{k}}$ are clearly disjoint. To prove a) it is enough to observe that we have

$$
\left(\mathcal{F}_{1}, \mathcal{F}_{2}, \kappa\right) \in G(\mathcal{B}) \backslash X_{C_{1}}^{\lambda_{1}} \cdots X_{C_{k}}^{\lambda_{k}} / G(\mathcal{B})
$$


if and only if there exists a chain

$$
\left(\mathcal{G}_{0}, \mathcal{G}_{1}, \kappa_{1}\right),\left(\mathcal{G}_{1}, \mathcal{G}_{2}, \kappa_{2}\right), \ldots,\left(\mathcal{G}_{k-1}, \mathcal{G}_{k}, \kappa_{k}\right) \in G(\mathcal{B}) \backslash G\left(\mathcal{O}_{\mathbf{F}}\right) / G(\mathcal{B})
$$

such that

(i) $\mathcal{G}_{0}=\mathcal{F}_{1}, \mathcal{G}_{k}=\mathcal{F}_{2}$;

(ii) $\kappa$ is equal to the composition of all the $\kappa_{j}$;

(iii) $\left(\mathcal{G}_{j-1}, \mathcal{G}_{j}, \kappa_{j}\right) \in G(\mathcal{B}) \backslash X_{C_{j}}^{\lambda_{j}} / G(\mathcal{B})$ for every $j=1, \ldots, k$.

This implies that $\kappa$ has singularities only on the curves $C_{1}, \ldots, C_{k}$ and the singularity of $\kappa$ at the generic point of $C_{j}$ is of type $\lambda_{j}$. In other words, this implies that

$$
\left(\mathcal{F}_{1}, \mathcal{F}_{2}, \kappa\right) \in G(\mathcal{B}) \backslash Y_{C_{1}, \ldots, C_{k}}^{\lambda_{1}, \ldots, \lambda_{k}} / G(\mathcal{B}),
$$

i.e. we have $X_{C_{1}}^{\lambda_{1}} \cdots X_{C_{k}}^{\lambda_{k}} \subset Y_{C_{1}, \ldots, C_{k}}^{\lambda_{1}, \ldots, \lambda_{k}}$. Thus a) follows from the fact that $X_{C_{1}, \ldots, C_{k}}^{\lambda_{1}, \ldots, \lambda_{k}} \subset Y_{C_{1}, \ldots, C_{k}}^{\lambda_{1}, \ldots, \lambda_{k}}$ and from the fact that all the $Y_{C_{1}, \ldots, C_{k}}^{\lambda_{1}, \ldots, \lambda_{k}}$ are disjoint.

Let us explain why Lemma 3.11 implies what we need. Let us fix a finite set $\mathcal{P}$ of collections $\left(\left(C_{1}, \lambda_{1}\right), \ldots,\left(C_{k}, \lambda_{k}\right)\right)$ with $C_{1}, \ldots, C_{k}$ pairwise distinct and with all $\lambda_{j} \in \Lambda_{+}$and $\lambda_{j} \neq 0$ if $k \neq 0$. Define a partial order on the set of all such collections by declaring that $\left(\left(C_{1}, \lambda_{1}\right), \ldots,\left(C_{k}, \lambda_{k}\right)\right) \geq$ $\left(\left(C_{1}^{\prime}, \lambda_{1}^{\prime}\right), \ldots,\left(C_{l}^{\prime}, \lambda_{l}^{\prime}\right)\right)$ if $k \geq l$ and after possible renumbering of the $C_{j}^{\prime}$ 's we have $C_{j}^{\prime}=C_{j}$ and $\lambda_{j}^{\prime} \leq \lambda_{j}$ for all $j=1, \ldots, l$. We shall assume that $\mathcal{P}$ is closed under the operation of replacing some $\left(\left(C_{1}, \lambda_{1}\right), \ldots,\left(C_{k}, \lambda_{k}\right)\right)$ by a smaller element (with respect to the above partial order). We want to show that the corresponding elements $\alpha_{C_{1}}\left(\lambda_{1}\right) \cdots \alpha_{C_{k}}\left(\lambda_{k}\right) \in \mathcal{H}_{\text {crit }}^{\text {sph }}$ are linearly independent.

Choose $n>0$ so that the assertions (2) and (3) hold for it. Consider now the corresponding elements $\alpha_{C_{1}}^{\mathbb{V}_{n}}\left(\lambda_{1}\right) \cdots \alpha_{C_{k}}^{\mathbb{V}_{n}}\left(\lambda_{k}\right)$. We claim that they are linearly independent as elements of $H\left(G_{n}, G_{n, \mathcal{O}}\right)$. Indeed, let us consider the restriction map from $H\left(G_{n}, G_{n, \mathcal{O}}\right)$ to the space $\mathcal{U}_{n}$ of $G_{n, \mathcal{O}}$-biinvariant functions on

$$
\bigcup X_{n, C_{1}, \ldots, C_{k}}^{\lambda_{1}, \ldots, \lambda_{k}}
$$

Let also $T_{n, C_{1}, \ldots, C_{k}}^{\lambda_{1}, \ldots, \lambda_{k}}$ be the characteristic function of $X_{n, C_{1}, \ldots, C_{k}}^{\lambda_{1}, \ldots, \lambda_{k}}$ and set $\mathcal{U}_{n, \mathcal{P}}$ to be the span of all the $T_{n, C_{1}, \ldots, C_{k}}^{\lambda_{1}, \ldots, \lambda_{k}}$ with $\left(\left(C_{1}, \lambda_{1}\right), \ldots,\left(C_{k}, \lambda_{k}\right)\right) \in \mathcal{P}$. Then it is clear from (3) above that they form a basis of $\mathcal{U}_{n, P}$. On the other hand, it follows from (1) and (2) that the images (in $\mathcal{U}_{n}$ ) of all the $\alpha_{C_{1}}^{\mathbb{V}_{n}}\left(\lambda_{1}\right) \cdots \alpha_{C_{k}}^{\mathbb{V}_{n}}\left(\lambda_{k}\right)$ lie in $\mathcal{U}_{n, \mathcal{P}}$ and that the transformation taking 
$T_{n, C_{1}, \ldots, C_{k}}^{\lambda_{1}, \ldots, \lambda_{k}}$ to the image $\alpha_{C_{1}}^{\mathbb{V}_{n}}\left(\lambda_{1}\right) \cdots \alpha_{C_{k}}^{\mathbb{V}_{n}}\left(\lambda_{k}\right)$ in $\mathcal{U}_{n}$ is upper triangular with respect to the partial order on $\mathcal{P}$ defined above with non-zeros on the diagonal. This shows that the elements $\alpha_{C_{1}}^{\mathbb{V}_{n}}\left(\lambda_{1}\right) \cdots \alpha_{C_{k}}^{\mathbb{V}_{n}}\left(\lambda_{k}\right)$ are linearly independent.

\subsection{Density conjecture}

The above results show that there exists an embedding $\mathcal{R}\left[\Lambda_{+}\right] \hookrightarrow \mathcal{H}_{\text {crit }}^{\mathrm{sph}}$. Though we do not expect this map to be an isomorphism we still would like to say that the left hand side is dense in the right hand side in some sense. One of the ways to do this is as follows.

Conjecture 3.13. For every $n \geq 0$ the images of $\mathcal{R}\left[\Lambda_{+}\right]$and of $\mathcal{H}_{\text {crit }}^{\text {sph }}$ in $\operatorname{End}\left(\mathcal{S}_{\text {crit }}\left(\hat{\mathbb{G}} / \Gamma_{n}\right)^{G(\mathcal{A})}\right)$ coincide.

This conjecture is obvious for $n=0$ (more precisely it follows from Lemma 3.8 that $\mathcal{S}_{\text {crit }}\left(\hat{\mathbb{G}} / \Gamma_{0}\right)=\mathcal{S}_{\text {crit }}\left(\hat{\mathbb{G}} / G\left(\mathcal{O}_{\mathbf{F}}\right)\right)=\mathbb{C}$ and hence there is nothing to prove). Also, it follows from Lemma 3.9 that Conjecture 3.13 is true for $n=1$. We do not know how to prove Conjecture 3.13 for $n>1$.

\subsection{The Iwahori case}

Let us briefly mention what happens in the Iwahori case. In other words we want to study the algebra $H_{c}\left(\hat{\mathbb{G}}, \mathbf{I}_{\mathcal{A}}^{00}\right)$ which is by definition the algebra of endomorphisms of the functor of $\mathbf{I}_{\mathcal{A}}$-invariants on the category $\operatorname{Rep}_{c}(\hat{\mathbb{G}})$. In this case we can't describe in full detail even a dense subalgebra in $H_{c}\left(\hat{\mathbb{G}}, \mathbf{I}_{\mathcal{A}}^{00}\right)$. However, from the results [8] and [4] one can derive the following (details will appear elsewhere):

(i) For any $C \in \mathcal{C}$ one can construct an embedding $\beta_{C}: \ddot{\mathbf{H}} \hookrightarrow H_{c}\left(\hat{\mathbb{G}}, \mathbf{I}_{\mathcal{A}}^{00}\right)$ where $\ddot{\mathbf{H}}$ denotes the modified Cherednik's double affine Hecke algebra as in [8].

(ii) There is a natural subalgebra of $H_{c}\left(\hat{\mathbb{G}}, \mathbf{I}_{\mathcal{A}}^{00}\right)$ isomorphic to $\mathbb{C}[\Lambda]$ which lies in the image of every $\beta_{C}$.

Recall that the set $\mathcal{C}$ only consists of good formal curves, i.e. curves which are transversal to the curve $t=0$. We expect that every irreducible curve in $\operatorname{Spec} \mathcal{B}$ (cf. Section 1.6) contributes some sort of subalgebra to $H_{c}\left(\hat{\mathbb{G}}, \mathbf{I}_{\mathcal{A}}^{00}\right)$. It would be extremely interesting to describe these subalgebras explicitly.

\section{$\S 4$. Some remarks on $G$-bundles on $S_{X}^{0}$}

In this section we would like to explain some constructions related to $G$-bundles on the "surface" $S_{X}^{0}$ that were used in Section 1.14. We are 
going to work over an arbitrary ground field $k$ (instead of the finite field $\mathbb{F}_{q}$ ). In what follows we fix the following data:

1) A smooth geometrically irreducible algebraic surface $S$ over $\mathbb{F}_{q}$;

2) A smooth projective geometrically irreducible curve $X$ over $\mathbb{F}_{q}$;

3) A closed embedding $i: X \rightarrow S$;

4) A "retraction" $p: S \rightarrow X$ such that $p \circ i=\mathrm{id}$. ${ }^{11}$

We denote by $\mathcal{I}_{X} \subset \mathcal{O}_{S}$ the sheaf of ideals corresponding to $X$.

Also we shall assume that the derived group of $G$ is simply connected.

\section{1. $G$-bundles on $S_{X}$}

Let us denote by $S_{X}$ the formal neighbourhood of $X$ in $S$ considered as an ind-scheme. In other words, we set

$$
S_{X, n}=\operatorname{Spec}_{\mathcal{O}_{X}}\left(\mathcal{O}_{S} / \mathcal{I}_{X}^{n}\right) \quad \text { and } \quad S_{X}=\lim _{\rightarrow} S_{X, n}
$$

By a $G$-bundle on $S_{X}$ we mean a projective system of $G$-bundles on $S_{X, n}$. In other words, to specify a $G$-bundle $\mathcal{G}$ on $S_{X}$ we need to specify a $G$-bundle $\mathcal{G}_{n}$ on each $S_{X, n}$ together with the isomorphisms

$$
\left.\mathcal{G}_{n}\right|_{S_{X, m}} \simeq \mathcal{G}_{m}
$$

for each $n \geq m$ satisfying the standard transitivity condition.

\section{2. $G$-bundles on $S_{X}^{0}$}

In Section 1.14 we were talking about $G$-bundles on $S_{X}^{0}$ where the latter was defined as the complement of $X$ in $S_{X}$. Let first explain what we mean by a $G$-bundle on $S_{X}^{0}$. Namely, we define the category of $G$-bundles on $S_{X}^{0}$ to be category whose objects are $G$-bundles on $S_{X}$ and morphisms are isomorphisms of their restrictions on $S_{X}^{0}$ (thus, by the definition, every $G$-bundle on $S_{X}^{0}$ extends to a $G$-bundle on $S_{X}$ ).

Warning. This definition is not good for families of $G$-bundles on $S_{X}^{0}$.

We denote by $\operatorname{Bun}_{G}\left(S_{X}^{0}\right)$ the set of isomorphism classes of $G$-bundles on $S_{X}^{0}$ with respect to the above definition.

\footnotetext{
${ }^{11}$ This datum is not probably not necessary.
} 


\subsection{Construction of the torsor $\widetilde{\operatorname{Bun}_{G}}\left(S_{X}^{0}\right)$}

Let us explain the construction of the torsor $\widetilde{\operatorname{Bun}}_{G}\left(S_{X}^{0}\right)$. Since in this paper we are constructing all the spaces only set-theoretically (i.e. we do not consider families) what we actually have to do is describe a $\operatorname{Pic}(X)$-torsor $\widetilde{\operatorname{Bun}}_{G}\left(S_{X}^{0}\right)_{\mathcal{M}}$ for every $\mathcal{M} \in \operatorname{Bun}_{G}\left(S_{X}^{0}\right)$.

Consider the following functor

$$
\text { Schemes over } \mathbb{F}_{q} \longrightarrow \text { Sets }
$$

which sends every scheme $T$ to the following data:

1) A morphism $T \rightarrow X$;

2) A G-bundle $\mathcal{G}$ on $T \times_{X} S_{X}$;

3) An isomorphism $\left.\mathcal{G}\right|_{T \times_{X} S_{X}^{0}} \simeq \operatorname{pr}_{2}^{*} \mathcal{M}$

where $\operatorname{pr}_{2}: T \times_{X} S_{X}^{0} \rightarrow S_{X}^{0}$ denotes the natural projection. This functor is representable by an ind-scheme $\operatorname{Gr}_{\mathcal{M}}$ endowed with a natural proper map $\gamma: \operatorname{Gr}_{\mathcal{M}} \rightarrow X$. It is easy to see that every fiber of $\gamma$ is non-canonically isomorphic to the corresponding affine Grassmannian of $\mathrm{Gr}_{G}$ of $G$ considered in Section 2. We now define the $\widetilde{\operatorname{Bun}_{G}}\left(S_{X}^{0}\right)_{\mathcal{M}}$ to be the set of isomorphism classes of line bundles $\mathcal{D}_{\mathcal{M}}$ on $\operatorname{Gr}_{\mathcal{M}}$ satisfying the following property:

For every $x \in X$ the restriction of $\mathcal{D}_{\mathcal{M}}$ to $\gamma^{-1}(x)$ is isomorphic to the generator $\mathcal{D}$ of $\operatorname{Pic}\left(\gamma^{-1}(x) \simeq \operatorname{Pic}\left(\operatorname{Gr}_{G}\right)\right.$.

Since $\mathcal{D}$ is defined uniquely up to non-canonical isomorphism and since its group of automorphisms is just the multiplicative group (since $\mathrm{Gr}_{G}$ is ind-proper) it follows that $\mathcal{D}_{\mathcal{M}}$ is defined uniquely up to tensoring with a line bundle of the form $\gamma^{*} \mathcal{P}$ where $\mathcal{P} \in \operatorname{Pic}(X)$. Hence $\widetilde{\operatorname{Bun}_{G}}\left(S_{X}^{0}\right)$ is a homogeneous space over $\operatorname{Pic}(X)$. Since the map $\gamma$ has sections (this is equivalent to saying that $\mathcal{M}$ extends from $S_{X}^{0}$ to $\left.S_{X}\right)$ it follows that $\widetilde{\operatorname{Bun}_{G}}\left(S_{X}^{0}\right)$ is actually a $\operatorname{Pic}(X)$-torsor.

\subsection{Proof of Lemma $\mathbf{1 . 1 5}$}

We need to construct an action of $\widetilde{\mathbb{G}}_{W}$ on $\widetilde{\operatorname{Bun}_{G}}\left(S_{X}^{0}, W\right)$. To simplify the notations let us do that for $\operatorname{Bun}_{G}$ instead of $\operatorname{Bun}_{G}$ (the general case is treated in a very similar manner). Let us denote by $S_{X \backslash W}^{0}$ the preimage of $X \backslash W$ in $S_{X}^{0}$. Then by arguing in a way similar to the one in the proof of Lemma 3.8 we see the group $\mathbb{G}_{W}$ may be identified with the set of 
isomorphism classes of $G$-bundles on $S_{X}^{0}$ trivialized at both $S_{W}^{0}$ and $S_{X \backslash W}^{0}$. Hence we have

$$
\operatorname{Bun}_{G}\left(S_{X}^{0}\right)=G\left(\mathcal{A}_{W}\right) \backslash \mathbb{G}_{W} / G\left(S_{X \backslash W}^{0}\right)
$$

and

$$
\operatorname{Bun}_{G}\left(S_{X}^{0}, W\right)=\mathbb{G}_{W} / G\left(S_{X \backslash W}^{0}\right) .
$$

The right hand side has now a natural action of $\mathbb{G}_{W}$.

\section{REFERENCES}

[1] A. Beilinson and V. Drinfeld, Quantization of Hitchin's integrable system and Hecke eigen-sheaves, preprint.

[2] P. Deligne, Extensions centrales de groupes algébriques simplement connexes et cohomologie galoisienne, Inst. Hautes Études Sci. Publ. Math., 84 (1996), 35-89.

[3] G. Faltings, Algebraic loop groups and moduli spaces of bundles, J. Eur. Math. Soc., 5 (2003), 41-68.

[4] D. Gaitsgory and D. Kazhdan, Representations of algebraic groups over a 2-dimensional local field, Geom. Funct. Anal., 14 (2004), no. 3, 535-574.

[5] D. Gaitsgory and D. Kazhdan, Algebraic groups over a 2-dimensional local field: some further constructions, math.RT/0406282.

[6] H. Garland, The arithmetic theory of loop groups. II. The Hilbert-modular case, J. Algebra, 209 (1998), no. 2, 446-532.

[7] M. Kapranov, The elliptic curve in the S-duality theory and Eisenstein series for Kac-Moody groups, math.AG/0001005.

[8] M. Kapranov, Double affine Hecke algebras and 2-dimensional local fields, J. Amer. Math. Soc., 14 (2001), no. 1, 239-262.

[9] S. Kumar, Demazure character formula in arbitrary Kac-Moody setting, Invent. Math., 89 (1987), no. 2, 395-423.

[10] O. Mathieu, Formules de caractères pour les algèbres de Kac-Moody générales, Astérisque No. 159-160, 1988, pp. 267.

[11] D. Quillen, Higher algebraic K-theory. I, Proc. Conf., Battelle Memorial Inst., Seattle, Wash., 1972, Lecture Notes in Math., Vol. 341, Springer, Berlin (1973), pp. 85-147.

Alexander Braverman

Einstein Institute of Mathematics

Edmond J. Safra campus, Hebrew University of Jerusalem

Givat-Ram, 91904 Jerusalem

Israel

braval@math. brown. edu 
David Kazhdan

Mathematics Department

Box 1917

Brown University

Providence, RI 02912

U.S.A.

kazhdan@math.huji.ac.il 